\title{
Symmetry-Breaking for Positive Solutions of Semilinear Elliptic Equations
}

\author{
Joel SMoller \& ARthur Wasserman
}

Communicated by J. SERRIN

\section{Introduction}

In a recent interesting paper, Gidas, NI, and NIRENBERG [2] proved that positive solutions of the Dirichlet problem for second-order semi-linear elliptic equations on balls must themselves be spherically symmetric functions. Here we consider the bifurcation problem for such solutions. Specifically, we investigate the ways in which the symmetric solution can bifurcate into a nonsymmetric solution; when this happens, we say that the symmetry "breaks." To carry out this program, we rely on certain results in [6], where we studied the kernel of the associated linearized operator. This enables us to give some necessary conditions for symmetry to break. We also find a class of functions $f$ where these conditions are also sufficient; see equation (18) below. The problem is, of course, to show that, when zero comes into the spectrum of the linearized operator and our conditions are fulfilled, bifurcation into the non-radial direction actually occurs. This is done by showing first that the "bifurcation curve" is a smooth manifold near the bifurcation point, and then appealing to a theorem of VANDERBAUWHEDE [8], which gives sufficient conditions for bifurcation to occur in the presence of symmetries.

\section{Degenerate Solutions}

We consider the Dirichlet problem

$$
\begin{aligned}
\Delta u(x)+f(u(x)) & =0, & & x \in D_{R}^{n} \\
u(x) & =0, & & x \in \partial D_{R}^{n} .
\end{aligned}
$$

Here $f \in C^{1}$ and $D_{R}^{n}$ denotes an $n$-ball of radius $R$. From the results in [2], all positive solutions of (1) are functions of the radius $r$ and satisfy the inequality $u_{r}(r)<0$, for $0<r<R$. Such solutions must therefore solve the boundary- 
value problem

$$
\begin{gathered}
u_{r r}+\frac{n-1}{r} u_{r}+f(u)=0, \quad 0<r<R, \\
u_{r}(0)=u(R)=0
\end{gathered}
$$

it is natural to think of these radial solutions of (1) as symmetric functions. Our purpose in this paper is to study how "symmetry" can be broken, that is, to describe the bifurcations of these positive symmetric solutions to non-radial solutions of (1).

If $u$ is a (positive) solution of (2), and $u(0)=p>0$, we write $u=u(r, p)$. Define

$$
A=\{p>0: u(r, p)=0 \text { for some } r>0\},
$$

and for $p \in A$, set

$$
T(p)=\min \{r>0: u(r, p)=0\} .
$$

The function $p \mapsto T(p)$, from $A$ into $\mathbb{R}_{+}$, will be called the time-map (see $\left.[4,5]\right)$. Clearly $u(r, p)$ is a solution of (2), (3) if and only if $T(p)=R$.

We say that a solution $u$ of (1) is non-degenerate provided that $v \equiv 0$ is the only solution of the linearized equations

$$
\begin{array}{rlrl}
\Delta v(x)+f^{\prime}(u(x)) v(x) & =0, & & x \in D_{R}^{n}, \\
v(x) & =0, & x \in \partial D_{R}^{n} .
\end{array}
$$

Thus $u$ is non-degenerate if and only if zero is not in the spectrum of the associated linearized operator; otherwise $u$ is called degenerate. It is not hard to show that a necessary condition for a solution to bifurcate is that it be degenerate.

In [6] the following characterization of non-degenerate (positive) solutions of (1) was obtained.

Theorem 1. Let $u=u(r, p)$ be a positive solution of (1). Then $u$ is nondegenerate if and only if

$$
u_{r}(T(p), p) \neq 0 \quad \text { and } \quad T^{\prime}(p) \neq 0 .
$$

In the proof it is shown that if $v$ is a solution of (4) corresponding to the positive solution $u(r, p)$, then, in terms of spherical harmonics, $v$ has the form

$$
v(r, \theta)=a_{0}(r)+a_{1}(r) \Phi(\theta), \quad 0 \leqq r \leqq R, \quad \theta \in S^{n-1}
$$

Here $\Phi$ is an eigenfunction of the Laplacian $\Delta$ on the $(n-1)$-sphere $S^{n-1}$, corresponding to the first non-zero eigenvalue. That is, all of the higher modes vanish identically. Since the corresponding eigenspace is isomorphic to the standard representation of the orthogonal group $O(n)$ on $\mathbb{R}^{n}$ (by $n \times n$ orthogonal matrices), and since $a_{0}$ and $a_{1}$ both satisfy linear second-order differential equations with linear boundary conditions, it follows that the kernel of the linearized operator is at most $(n+1)$-dimensional. That is, $a_{0}$ and $a_{1}$ are unique up to a constant multiple, and the set of $\Phi$ 's is $n$-dimensional. 
From the results in [6] we know that $a_{1}$ is zero if and only if

$$
u_{r}(T(p), p) \neq 0 \text {, }
$$

and $a_{0}$ is zero if and only if

$$
u_{r}(T(p), p) T^{\prime}(p) \neq 0 .
$$

We summarize these results in the following theorem.

Theorem 2. Any solution of (4) relative to a positive solution $u(r, p)$ of (1) has the form (6). The function $a_{0}$ is zero if and only if (8) holds, while $a_{1}$ is zero if and only if (7) holds.

\section{Symmetry Breaking}

It is natural to parametrize positive radial solutions of (2), (3) by $p=u(0, p)$, this being the maximum of $u(r, p)$ since $u^{\prime}<0$. Now suppose that we have a family of radial solutions $u(r, p), p_{1} \leqq p \leqq p_{2}$.

Definition 1. Let $0<p_{1}<\bar{p}<p_{2}$. Then the symmetry is said to break infinitesimally at $u(\cdot, \bar{p})$ provided there exists a solution of (4) with $a_{1} \neq 0$; see (6).

2. The symmetry is said to break at $u(\cdot, \bar{p})$ provided that a non-radial solution bifurcates out of $u(\cdot, \bar{p})$.

It is not difficult to show that symmetry breaking implies infinitesimal symmetry breaking; see for example [1]. We investigate first the weaker notion.

In view of the discussion in the last section, we may assume that, for some $\bar{p}, p_{1}<\bar{p}<p_{2}$,

$$
u_{r}(T(\bar{p}), \bar{p})=0 .
$$

Let $K$ denote the kernel of the associated linearized operator, namely the operator

$$
\Delta+f^{\prime}(u(r, \bar{p})) \quad \text { on }\left\{u \in C^{2}\left(D_{T(\bar{p})}^{n}\right): u\left(\partial D_{T(\bar{p})}^{n}\right)=0\right\} .
$$

Define the set

$$
B=\left\{\partial u(r, \bar{p}) / \partial x_{i}: 1 \leqq i \leqq n\right\}
$$

We then have the following result.

Theorem 3. (a) Assume that (9) holds; then the elements of $B$ are linearly independent and lie in $K$.

(b) No element in $B$ is a radial function.

(c) The action of the orthogonal group $O(n)$ on the space spanned by $B$ is irreducible (in the sense that it has no proper invariant subspaces).

Proof. Let $v$ be any non-zero vector in $\mathbb{R}^{n}$, and let $D_{v}$ denote differentiation in the direction $v$. Since $D_{v}$ commutes with $\Delta$, we see that $D_{v} u(r, \bar{p})$ satisfies the 
linearized equations. Since $u$ is constant on the sphere we have $D_{v} u=0$ if $\langle v, p\rangle=0$, while also $D_{v} u(T(\bar{p}), \bar{p})=0$ in view of (9). It follows that $B C K$. If $D_{v} u(r, \bar{p}) \equiv 0$, then $u$ would be constant in the $v$ direction, and then since $u(T(\bar{p}), \bar{p})=0$ we would have $u(r, \bar{p})=0,0 \leqq r \leqq T(\bar{p})$. This violates the condition $u(0, \bar{p})=\bar{p}>0$; thus (a) holds. Now

$$
\frac{\partial u}{\partial x_{i}}=\frac{x_{i}}{r} u_{r}
$$

and this shows that $\partial u / \partial x_{i}$ is non-radial for each $i$; this proves (b). For (c), let $T \in O(n)$. Then for any $v \in \mathbb{R}^{n}$ we have

$$
T D_{v} u(r, \bar{p})=D_{T v} u(r, \bar{p}) .
$$

It follows that the action of $O(n)$ on $B$ is the same as the standard action of $O(n)$ on $\mathbb{R}^{n}$, and thus is irreducible. The proof of the theorem is now complete.

We shall next obtain a necessary condition for infinitesimal (and hence actual) symmetry breaking.

Theorem 4. For positive solutions, infinitesimal symmetry breaking cannot occur if $f(0) \geqq 0$.

Proof. Write (2) as the first order system

$$
u_{r}=v, \quad v_{r}=-\frac{n-1}{r} v-f(u) .
$$

If infinitesimal symmetry breaking occurs, then (11) has a positive solution with $u(R)=0=v(R)$, for some $R>0$. (Recall from [2] that $v(r)<0$ if $0<r<R$.) At this point $R$ we have $u_{r}=0$ and $v_{r}>0$; see Figure 1 . Thus from (11) we find that $v_{r}(R)=-f(u(R))=-f(0)$. Formally, if $f(0)>0$ then $v_{r}(R)<0$, and so $v(R-\varepsilon)>0$ for some $\varepsilon>0$. Hence $f(0) \leqq 0$. If $f(0)=0$, then $(0,0)$ is a "rest point" and no orbit enters $(0,0)$ in finite time. Therefore $f(0)<0$. (Notice that $u_{r}=v>0$ when $r>R$ and $r-R$ is small, so that the orbit in Figure 1 actually breaks into the region $\{u>0\}$, as depicted.)

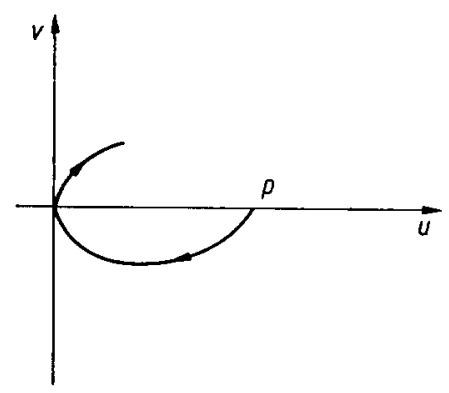

Fig. 1 
We have thus shown that if the symmetry breaks at $u(r, \bar{p})$, then necessarily

$$
f(0)<0
$$

and in addition

$$
u(\bar{R}, \bar{p})=u_{r}(\bar{R}, \bar{p})=0 .
$$

The last condition implies that the function $u(r, \bar{p})$ is a solution of both the Dirichlet and Neumann problems on the ball $\{0 \leqq r \leqq \bar{R}\}$. We now consider the question of primary interest; if (13) holds (so that (12) also holds), does the symmetry break at $u(r, \bar{p})$ ?

As is easily seen from simple examples, bifurcation need not occur even if the kernel of the linearized operator is non-zero; that is, control of the first-order terms does not necessarily imply that there is actual bifurcation. Some condition on the higher-order terms is also needed. Thus, for example in the proof of the Crandall-Rabinowitz Theorem (see [7]), one must impose a certain "transversality" hypothesis, taking the form of a condition on a mixed derivative. In the case considered here, in order to prove that bifurcation actually occurs, we shall appeal to an extension of the Crandall-Rabinowitz Theorem due to VANDERBAUWHEDE [8, Theorem 6.2.6]. This result applies when the kernel of the linearized operator has dimension greater than one, and when this kernel is also invariant under the action of the orthogonal group $O(n)$. We now state the theorem in a form suitable to our purposes.

Let $X, Z$ and $A$ be real Banach spaces, and let $M$ be a mapping of class $C^{2}$ of $\Omega$, a neighborhood of the origin in $X \times \Lambda$, into $Z$. We assume that $M(0,0)=0$, and that the following three hypotheses hold.

$\left(\mathrm{H}_{1}\right)$ There exist representations $\Gamma: O(n) \rightarrow L(X)$ and $\tilde{\Gamma}: O(n) \rightarrow L(Z)$ of the orthogonal group (where $L(X)$ and $L(Z)$ denote the set of linear transformations on $X$ and $Z$, respectively), such that $M$ is equivariant with respect to $(O(n), \Gamma, \tilde{\Gamma})$. That is, for each $s \in O(n)$ and all $(x, \lambda) \in \Omega$, we have

(i) $(\Gamma(s) x, \lambda) \in \Omega$, and

(ii) $M(\Gamma(s) x, \lambda)=\tilde{\Gamma}(s) M(x, \lambda)$.

$\left(\mathrm{H}_{2}\right) L \equiv D_{x} M(0,0)$ is a Fredholm operator of index zero, and the representation $\Gamma_{\mathrm{o}}$ of $O(n)$ induced by $\Gamma$ on $\operatorname{ker} L$ is irreducible.

$\left(\mathrm{H}_{3}\right)$ There is a non-zero vector $u_{0}$ in $\operatorname{ker} L$ such that $D_{\lambda} D_{x} M(0,0) \cdot\left(0, u_{0}\right) \notin$ Range $(L)$.

Under these hypotheses, VANDERBAUWHEDE's Theorem states that $(0,0)$ is a bifurcation point for the equation $M(x, \lambda)=0$. We shall now apply this theorem to our problem.

As above, we assume that (1) has positive radial solutions $u(r, p)$ on some interval $p_{1} \leqq p \leqq p_{2}$. For such $p$ we consider the equation

$$
u(R, p)=0 .
$$

Suppose that $u(T(\vec{p}), \bar{p})=0$ for some $\bar{p}, p_{1} \leqq \bar{p} \leqq p_{2}$ and

$$
u_{p}(T(\bar{p}), \bar{p}) \neq 0 \text {. }
$$


The implicit function theorem then implies that we may write $p=p(R)$ near the point $(T(\bar{p}), \bar{p})$. In this case, from the equation $u(T(p), p)=0$ we obtain

$$
u_{r}(T(p), p) T^{\prime}(p)+u_{p}(T(p), p)=0,
$$

and thus $\left|T^{\prime}(\bar{p})\right|=\infty$ as well as

$$
p^{\prime}(\bar{R})=0, \quad \text { where } \quad \bar{R}=T(\bar{p}) .
$$

We illustrate this situation in Figure 2 (a), where the branch $\bar{R} \geqq R \geqq \bar{R}-\varepsilon$ consists of positive solutions while the other branch is made up of non-positive solutions; cf. Figure 2(b).
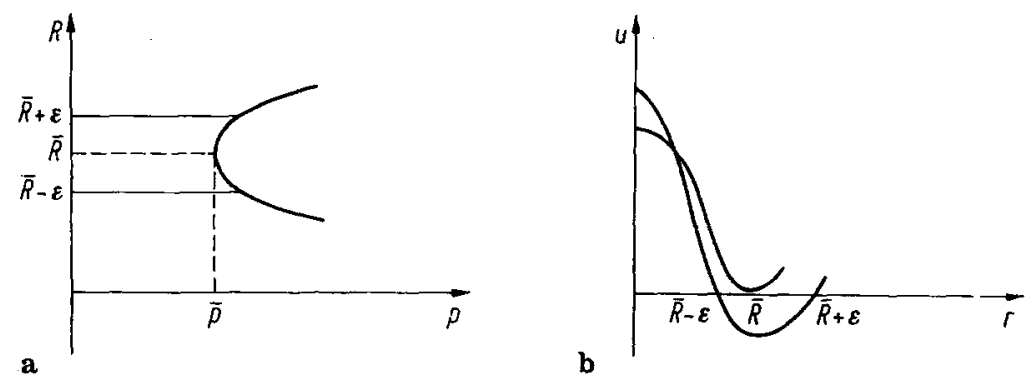

Fig. 2

Still assuming (14), we define the operator

$$
M:\left\{u \in C^{2}(|x|<1): u(x)=0 \text { if }|x|=1\right\} \times I \rightarrow\{u \in C(|x|<1)\}
$$

by

$$
M(z, R)(x)=\Delta(z(x)+u(|x| R, p(R)))+R^{2} f(z(x)+u(|x| R, p(R))),
$$

where $I=[0,1] u(r, p(R))$ is a radial solution of (1) and $\bar{R}-\varepsilon \leqq R \leqq \bar{R}+\varepsilon$, $0 \leqq R \leqq 1$. Note that $M(0, R)=0$ and that $M$ is equivariant * with respect to the natural action of $O(n)$. Furthermore, $M_{z}(0, R)$ is a Fredholm operator of zero index, as follows from standard results; see [3]. Finally, Theorem 3 shows that the induced representation of $O(n)$ on the kernel of $M_{z}(0, R)$ is irreducible. We have thus verified hypotheses $\left(\mathrm{H}_{1}\right)$ and $\left(\mathrm{H}_{2}\right)$ of VANDERBAUWHEDE's Bifurcation Theorem. In order to verify the transversality hypothesis $\left(\mathrm{H}_{3}\right)$, we compute

$$
\begin{aligned}
& M_{Z R}(Z, R) \\
& \quad=R^{2} f^{\prime \prime}(z+u(r R, p(R)))\left[u^{\prime}(r R, p(R)) r+u_{p} p^{\prime}(R)\right]+\frac{2}{R} f^{\prime}(z+u(r R, p(R))),
\end{aligned}
$$

so that in view of (15)

$$
M_{Z R}(0, \bar{R})=\bar{R}^{2}\left\{f^{\prime \prime}(u(r \bar{R}, \bar{p})) u^{\prime}(r \bar{R}, \bar{p}) r+2 \bar{R}^{-1} f^{\prime}(u(r \bar{R}, \bar{p}))\right\}
$$

* $M(Z \circ T, R)(x)=M(Z, R)(T x)$ for all $T \in O(n)$. 
The crucial transversality hypothesis can now be stated as

$$
\int_{|x|<1} M_{Z R}(0, \bar{R}) v^{2}(x) d x \neq 0
$$

for some $v$ in the kernel of $M_{z}(0, \bar{R})$. From [2] we know that $u_{r}(r \bar{R}, \bar{p}) \leqq 0$ in $0 \leqq r \leqq 1$; thus (17) holds if $f^{\prime}$ and $f^{\prime \prime}$ have the opposite sign. We have therefore proved the following result.

Theorem 5. Suppose that (14) holds and that the symmetry breaks infinitesimally at $\bar{p}$. Then, if $f^{\prime}$ and $f^{\prime \prime}$ have the same sign, the symmetry breaks at $\bar{p}$.

In order to apply this theorem we must still investigate the conditions under which (14) holds. As we shall show below, any $C^{2}$ function $f$ can be composed with an arbitrarily small translation so that (14) holds. Thus, for example, let

$$
f(u)=A u-B+e^{-u}, \quad A>0, B>0,
$$

where $A$ and $B$ are constants. Note that $f(0)<0$ and that $f^{\prime}<0$ and $f^{\prime \prime}>0$; thus (17) will always hold for the functions (18).

To verify (14), we consider $u=u(r, p)$ as a $C^{3}$ function ${ }^{\star}$ of its arguments. If $u$ is a radial solution of (1), then of course $u(R, p)=0$. Suppose now that 0 is a regular value of $u$ (see [7]). Then

$$
\left(u_{r}(R, p), u_{p}(R, p)\right) \neq(0,0) .
$$

Hence the condition $u_{r}(\bar{R}, \bar{p})=0$ implies that $u_{p}(\bar{R}, \bar{p}) \neq 0$, so (14) holds. Now by SARD's Theorem [7] the set of critical values of a smooth function has measure zero. Thus, let $\delta>0$ be a regular value of $u$ and set

Then

$$
\tilde{u}=u-\delta, \quad g(\tilde{u})=f(u) .
$$

$$
\Delta \tilde{u}+g(\tilde{u})=\Delta u+f(u),
$$

and $\tilde{u}=0$ if and only if $u=\delta$. Also, using (18), we have

$$
g(\tilde{u})=e^{-\delta}\left[A e^{\delta} \tilde{u}-e^{\delta}(B-A \delta)-e^{-\tilde{u}}\right] .
$$

Thus we set

$$
A^{\prime}=A e^{\delta}, \quad B^{\prime}=e^{\delta}(B-A \delta),
$$

so that $A^{\prime}, B^{\prime}>0$ small $\delta$. Moreover, it is easily verified that the problem

$$
\Delta \tilde{u}+g(\tilde{u})=0 \quad \text { in } D_{R}^{n}, \quad \tilde{u}=0 \quad \text { on } \partial D_{R}^{n},
$$

has a solution if and only if the problem

$$
\Delta \tilde{u}+\operatorname{cg}(\tilde{u})=0 \quad \text { in } D_{\tilde{R}}^{n}, \quad \tilde{u}=0 \quad \text { on } \partial D_{\tilde{R}}^{n},
$$

has a solution, where $\tilde{R}=R / \sqrt{c}$. Thus, we can find a positive number $\delta_{0}$ such that (14) holds for almost $\delta$ such that $0 \leqq \delta \leqq \delta_{0}$.

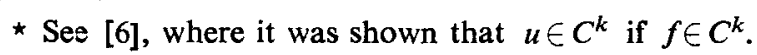


It was shown in [6, Corollary 14], that if $f$ grows linearly at infinity, that is, if $f(u)=O(u)$ as $u \rightarrow \infty$, then (1) has positive radial solutions $u=u(r, p)$ for all sufficiently large $p$. Note also that, for $f$ given by (18), we have $u f^{\prime}(u)>f(u)$. It follows from this (see [6, Proof of Lemma C]) that the set of points $p$ where $u_{r}(R, p)=0$ must be discrete. We shall now show that this set is non-empty.

To do this, we define

$$
\tilde{p}=\inf \{p: \tilde{u}(\cdot, p) \text { solves }(19)\} .
$$

Note that $\tilde{p}>p_{0}>0$, where $p_{0}$ is the unique positive root of $f(\mathrm{cf}$. (18)). We claim that the solution curve of (2) which satisfies $u(0)=\tilde{p}, u_{r}(0)=0$ passes through the origin, that is, $\left(u(R), u_{r}(R)\right)=(0,0)$ for some positive $R$. Indeed, observe that this orbit cannot meet $u=0$ at a point where $u_{r}<0$, nor can it meet $u_{r}=0$ at a point where $u>0$, since both such occurrences would imply the persistence of this behavior in a neighborhood of $\tilde{p}$, thus violating the definition of $\tilde{p}$. The only other possibility is that this orbit enters the point $\left(p_{0}, 0\right)$ infinite "time", that is,

$$
\lim _{r \rightarrow \infty}\left(u(r, \tilde{p}), u_{r}(r, \tilde{p})\right)=\left(p_{0}, 0\right) .
$$

To see that this too cannot happen, let $H$ be the "total energy",

$$
H\left(u, u_{r}\right)=\left(u_{r}\right)^{2} / 2+F(u) \quad\left(\text { where } F^{\prime}=f\right) .
$$

Then $H^{\prime}=-(n-1)\left(u_{r}\right)^{2} r^{-1}$, so $H$ decreases along solution curves. Moreover $H\left(p_{0}, 0\right)<0$. Thus if $(20)$ were true, then there would be some $R>0$ for which $H\left(u(r, \tilde{p}), u_{r}(r, \tilde{p})\right)<0$ if $r \geqq R$. We could then find a neighborhood $N$ of $(\tilde{p}, 0)$ such that all orbits of (2) starting in $N$ would have $H<0$ if $r \geqq 2 R$. But then no such orbit could be a positive solution of the Dirichlet problem, and this would again violate the definition of $\tilde{p}$. Our claim is now proved.

It follows that the symmetry breaks infinitesimally for all such solutions $u(r, \tilde{p})$. Hence if $f$ is given by (18), then the symmetry breaks for almost all $A$ and $B$ satisfying $A<0, B>1$. We have thus proved

Theorem 6. Consider the Dirichlet problem (1), where $f$ is given by (18). Then for almost all choices of $A$ and $B$ such that $A>B>1$ there are positive radial solutions for which the symmetry breaks.

For functions of the type (18) the bifurcation diagram near $\ddot{p}$ therefore has the form shown in Figure 3, namely there is a $n$-manifold of non-radial (hence non-positive) solutions which bifurcate out of $u(r, \tilde{p})$.

We close with a conjecture about symmetry breaking for a different class of functions $f$. Suppose in particular that $f=f(u)$ satisfies the conditions

$$
(f(u) / u)^{\prime}>0, \quad f^{\prime \prime}(u) \leqq 0, \quad f(0)<0 .
$$

For such functions it was shown in [5] that there is always a unique positive solution of (1) for which the symmetry breaks infinitesimally; our conjecture is that the symmetry must also actually break. 


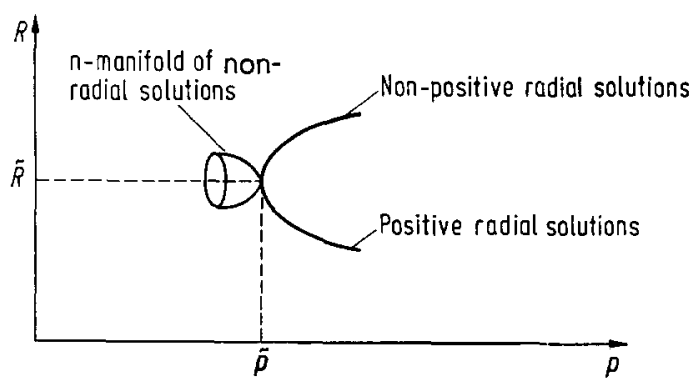

Fig. 3

Acknowledgement. The research of the first author was partially supported by the National Science Foundation under Contract No. MCS 83-01243.

\section{References}

1. Dancer, E. N., On non-radially symmetric bifurcation, J. London Math. Soc., 20 (1979), 287-292.

2. Gidas, B., W. M. Ni \& L. Nirenberg, Symmetry of positive solutions of nonlinear elliptic equations in $\mathbb{R}^{n}$, Comm. Math. Phys., 68 (1979), 209-243.

3. Palais, R., Seminar on the Atiyah-Singer Theorem, Annals of Math. Studies, No. 57, Princeton Univ. Press: Princeton 1965.

4. Smoller, J., \& A. Tromba \& A. Wasserman, Nondegenerate solutions of boundary-value problems, J. Nonlinear Anal., 4 (1980), 207-215.

5. Smoller, J., \& A. Wasserman, Global bifurcation of steady-state solutions, J. Diff. Equations 39 (1981), 269-290.

6. Smoller, J., \& A. Wasserman, Existence, uniqueness, and non-degeneracy of positive solutions of semilinear elliptic equations, Comm. Math. Phys., 95 (1984), 129-159.

7. Smoller, J., Shock Waves and Reaction-Diffusion Equations, Springer-Verlag: New York, 1983.

8. Vanderbauwhede, A., Local Bifurcation and Symmetry, Research Notes in Math., No. 75, Pitman: Boston, 1982.

\section{Mathematics Department University of Michigan Ann Arbor}

